\title{
An Overlapping Window Decorrelating Multiuser Detector for DS-CDMA Radio Channels
}

\author{
Xiafeng Wang, Student Member, IEEE, Wu-Sheng Lu, Fellow, IEEE, and Andreas Antoniou, Fellow, IEEE
}

\begin{abstract}
A new multiuser detector for direct-sequence codedivision multiple-access communication systems is described. The proposed detector divides the received signal stream into a number of overlapping windows and decorrelates them window by window. This scheme is justified by an analysis on the convergence and decay rate of the impulse response. Based on the results, a signaladapted criterion is developed which enables one to determine the window length according to the near-far situation of a practical system. A performance analysis and simulation results show that a small to moderate window length is usually sufficient to yield a performance that is close to or even better than that of the ideal decorrelating detector.
\end{abstract}

Index Terms-CDMA, decorrelation, multiuser detection.

\section{INTRODUCTION}

$\mathbf{T}$ HE prohibitive computational complexity of the optimal multiuser detector [1] has motivated the search for suboptimal multiuser detectors with much reduced computational complexity. As a result, a large number of suboptimal multiuser detectors were developed (see [2] and the references therein). Among these, the ideal decorrelating detector [3] achieves the near-far (NF) resistance of the optimal multiuser detection without the need for knowledge of the received signal energies. However, the ideal decorrelating detector involves computing and updating the inverse of the cross-correlation matrix, which require a considerable amount of computation. Furthermore, the detection delay of the ideal decorrelating detector for asynchronous systems is unacceptally large.

To achieve a practical detection delay, a window approach for implementing the ideal decorrelating detector was first suggested in [4], and a number of window-based decorrelating detectors were developed recently [5]-[7]. A problem related to these window-based decorrelating detectors is the edge effect caused by the multiple-access interference (MAI) from the transmitted signals outside the working window, where the working window is the window that is currently being processed. In [5] and [7], the edge effect is corrected at the expense of bandwidth efficiency or complexity of network management. In [6], the so-called truncated decorrelating detector

Paper approved by G. E. Corazza, the Editor for Spread Spectrum of the IEEE Communications Society. Manuscript received September 29, 1998; revised May 16, 2000. This paper was presented in part at the ICASSP, Seattle, WA, May 1998.

$\mathrm{X}$. Wang was with the Department of Electrical and Computer Engineering, University of Victoria, Victoria, BC V8W 3P6, Canada. He is now wit PMC-Sierrra Corporation, Burnaby, BC V5A 4V7, Canada (e-mail: xiaofeng_wang@pmc-sierra.com).

W.-S. Lu and A. Antoniou are with the Department of Electrical and Computer Engineering, University of Victoria, Victoria, BC V8W 3P6, Canada (e-mail: wslu@ece.uvic.ca; andreas@ece.uvic.ca).

Publisher Item Identifier S 0090-6778(01)06936-7. was proposed, whose performance can be arbitrarily close to that of the ideal decorrelating detector if the window length is sufficiently large. Another interesting window-based decorrelating detector was developed using a chip-rate approach, which leads to an exact finite-duration impulse response (FIR) solution and avoids the edge effects [8]. Note, however, that the bit-rate approach has been widely adopted in the literature for problems where user codes are known. This is because an exact decorrelating solution does not necessarily imply a satisfactory performance and issues concerning practical implementation are easier to handle in the bit-rate approach. The various approaches entail similar problems concerning practical implementation. These include (a) a matrix of large size needs to be inverted and (b) an appropriate window (or filter) length needs to be determined to assure a satisfactory performance with moderate computational complexity.

In this paper, we propose a new window-based detector, referred to as the overlapping window decorrelating (OWD) detector in which the aforementioned problems are easier to solve. Furthermore, an analysis of the convergence and decay properties of the ideal decorrelating impulse response is given. Although some of our analysis results have been noted in the past [4], [6] and can be related to those in [9], they provide further insight to the problem at hand and lead to a signal-adapted criterion for window-length determination. Based on the near-far situation of the system of interest, the signal-adapted criterion enables us to determine the minimum window length required for satisfactory performance. An analysis and computer simulations are carried out which show that a small to moderate window length is sufficient to provide performance close to or even better than that of the ideal decorrelating detector.

\section{System Model AND Multiuser Decorrelating DETECTION}

We consider multiuser transmission through an additive white Gaussian noise (AWGN) channel shared by $K$ asynchronous users in a DS-CDMA system. For the sake of simplicity, binary phase-shift-keying (BPSK) modulation is assumed but the results can be readily extended to the case of the more commonly used quadrature-phase shift-keying (QPSK) modulation. Let the message length be $N$ and denote the $k$ th information bit of the $i$ th user, the corresponding signal energy, and the output of the matched filter by $b_{i}(k), e_{i}(k)$, and $r_{i}(k)$, respectively. For coherent reception, the concatenation of $N$ successive output vectors of the $K$ matched filters, $\mathbf{r}$, can be modeled as [4]

$$
\mathbf{r}=\mathbf{R}_{N} \mathbf{E b}+\mathbf{n}
$$


where $\mathbf{r}=\left[\begin{array}{lll}\mathbf{r}^{\mathrm{T}}(1) & \mathbf{r}^{\mathrm{T}}(2) \cdots \mathbf{r}^{\mathrm{T}}(N)\end{array}\right]^{\mathrm{T}}, \mathbf{r}(k)=\left[r_{1}(k)\right.$ $\left.r_{2}(k) \cdots r_{K}(k)\right]^{\mathrm{T}}, \mathbf{b}=\left[\begin{array}{ll}\mathbf{b}^{\mathrm{T}}(1) & \mathbf{b}^{\mathrm{T}}(2) \cdots \mathbf{b}^{\mathrm{T}}(N)\end{array}\right]^{\mathrm{T}}, \mathbf{b}(k)=$ $\left[b_{1}(k) \quad b_{2}(k) \cdots b_{K}(k)\right]^{\mathrm{T}}, \mathbf{E}=\operatorname{diag}\{\mathbf{E}(1), \cdots, \quad \mathbf{E}(N)\}$, $\mathbf{E}(k)=\operatorname{diag}\left\{\sqrt{e_{1}(k)}, \sqrt{e_{2}(k)}, \cdots, \quad \sqrt{e_{K}(k)}\right\}$, and $\mathbf{n}$ is the AWGN vector with zero mean and covariance matrix $N_{0} \mathbf{R}_{N} / 2$. The correlation matrix $\mathbf{R}_{N}$, given in (2) at the bottom of the page, is an $(N \times N)$-block 3-band matrix. In (2), each $\mathbf{H}_{m}(k)$ for $m=0,1$ is a $K \times K$ matrix whose $(i, j)$ th entry is given by

$$
\begin{gathered}
h_{m, i j}(k)=\int_{-\infty}^{\infty} g_{i}^{k}\left(t-\tau_{i}\right) g_{j}^{k-1}\left(t+m T-\tau_{j}\right) d t, \\
\text { for } m=0,1
\end{gathered}
$$

where $g_{i}^{k}(t)$ denotes the normalized signature signal of the $k$ th bit of the $i$ th user and is zero outside interval $[0, T]$, and $\tau_{i}$ denotes the transmission delay for the $i$ th user. Without loss of generality, we arrange the transmission delays in ascending order, i.e., $0 \leq \tau_{1} \leq \cdots \leq \tau_{K}<T$, so as to obtain an upper triangular matrix $\mathbf{H}_{1}(k)$ for $1 \leq k \leq N$. In the rest of the paper, we will refer to a system as time invariant or time dependent depending on whether the correlation $\mathbf{H}_{m}(k)$ in the system remains constant or not with time.

Note that the above model also applies to a multirate system in which the largest permissible spreading factor is a multiple of all other smaller allowable spreading factors. In this case, a user with a smaller spreading factor can be deemed to be a number of virtual users. The virtual users associated with a real user have identical signature signals but occupying different time slots in one symbol interval.

A decorrelating multiuser detector can be described by

$$
\hat{\mathbf{b}}=\mathbf{E b}+\mathbf{R}_{N}^{-1} \mathbf{n}
$$

with the final bit estimates taken as sign $[\hat{\mathbf{b}}]$. Evidently, the ideal decorrelating detector requires no information about the received amplitudes and achieves optimal near-far resistance [4]. The estimate $\hat{\mathbf{b}}$ is the exact decorrelated data plus noise and the MAI is completely eliminated. An undesirable feature of this detector is that noise is always enhanced.

\section{OVERLAPPING Window DeCorRelating Detector}

\section{A. OWD Detector}

Within a window of length $M=2 p+1$, (1) can be written as

$$
\mathbf{r}_{i}-\mathbf{r}_{b}-\mathbf{r}_{e}=\mathbf{R}_{M} \mathbf{E}_{i} \mathbf{b}_{i}+\mathbf{n}_{i}
$$

where

$$
\begin{aligned}
\mathbf{b}_{i} & =\left[\mathbf{b}^{\mathrm{T}}(i-p) \cdots \mathbf{b}^{\mathrm{T}}(i) \cdots \mathbf{b}^{\mathrm{T}}(i+p)\right]^{\mathrm{T}} \\
\mathbf{r}_{i} & =\left[\mathbf{r}^{\mathrm{T}}(i-p) \cdots \mathbf{r}^{\mathrm{T}}(i) \cdots \mathbf{r}^{\mathrm{T}}(i+p)\right]^{\mathrm{T}} \\
\mathbf{r}_{b} & =\left\{\left[\mathbf{H}_{1}(i-p-1) \mathbf{E}(i-p-1) \mathbf{b}(i-p-1)\right]^{\mathrm{T}} 0 \cdots 0\right\}^{\mathrm{T}} \\
\mathbf{r}_{e} & =\left[0 \cdots 0 \mathbf{b}^{\mathrm{T}}(i+p+1) \mathbf{E}(i+p+1) \mathbf{H}_{1}(i+p)\right]^{\mathrm{T}} \\
\mathbf{E}_{i} & =\operatorname{diag}\{\mathbf{E}(i-p), \cdots, \mathbf{E}(i), \cdots, \mathbf{E}(i+p)\} .
\end{aligned}
$$

In (4), $\mathbf{n}_{i}$ represents Gaussian noise and $\mathbf{R}_{M} \in \mathcal{R}^{M K \times M K}$ is a submatrix of $\mathbf{R}_{N}$, which is also given by (2) except that index $k$ in $\mathbf{H}_{m}(k)$ assumes values from $i-p$ to $i+p$ instead of from 1 to $N$. It follows that within the window, $\mathrm{b}_{i}$ can be estimated as

$$
\tilde{\mathrm{b}}_{i}=\mathbf{R}_{M}^{-1}\left(\mathrm{r}_{i}-\mathrm{r}_{b}-\mathbf{r}_{e}\right)
$$

which requires knowledge of $\mathbf{r}_{b}$ and $\mathbf{r}_{e}$. The MAI at the beginning of the window $\mathbf{r}_{b}$ can be approximated by using the soft outputs $\tilde{\mathbf{b}}(i-p-1)$ whereas the MAI at the end of the window $\mathbf{r}_{e}$ is not available within the window. The OWD detector is deduced from (5) by neglecting term $\mathbf{r}_{e}$ and estimating the user information bits as

$$
\hat{\mathbf{b}}_{i}=\mathbf{R}_{M}^{-1} \tilde{\mathbf{r}}_{i}
$$

where

$$
\tilde{\mathbf{r}}_{i}=\mathbf{r}_{i}-\mathbf{r}_{b}
$$

In order to avoid an inaccurate estimate caused by neglecting $\mathbf{r}_{e}$, we take an approach whereby a window is overlapped by the next window in $p$ transmission intervals. Consequently, only the first $p+1$ bits in a working window need to be detected. As will be shown below, the impulse response of the ideal decorrelating detector usually decays very quickly; hence if the window length $M$ is sufficiently large, the effect from the MAI term $\mathbf{r}_{e}$ on the detection of user information bits at the first $p+1$ transmission intervals is negligible. Moreover, if an incorrect estimate of $\mathbf{r}_{b}$ is used in (7), the effect of the resulting left-edge correction on the detection of $\mathbf{b}(i)$ is also negligible. Since only $\tilde{\mathbf{b}}(i)$ is used for the left-edge correction in the next working window, the error caused by an incorrect left-edge correction will not propagate.

For efficient implementation of the proposed detector as well as later analysis, the Cholesky decomposition

$$
\mathbf{R}_{N}=\mathbf{L L}^{\mathrm{T}}
$$

$$
\mathbf{R}_{N}=\left[\begin{array}{cccccc}
\mathbf{H}_{0}(1) & \mathbf{H}_{1}^{\mathrm{T}}(1) & 0 & \ldots & \cdots & 0 \\
\mathbf{H}_{1}(1) & \mathbf{H}_{0}(2) & \mathbf{H}_{1}^{\mathrm{T}}(2) & 0 & \cdots & 0 \\
\vdots & \vdots & \vdots & \vdots & \vdots & \vdots \\
0 & 0 & 0 & \mathbf{H}_{1}(N-2) & \mathbf{H}_{0}(N-1) & \mathbf{H}_{1}^{\mathrm{T}}(N-1) \\
0 & 0 & 0 & 0 & \mathbf{H}_{1}(N-1) & \mathbf{H}_{0}(N)
\end{array}\right]
$$


where $\mathbf{L}$ is a lower triangular matrix, will be used. From (2) and (8), it can be verified that $\mathbf{L}$ has the form

$$
\mathbf{L}=\left[\begin{array}{cccccc}
\mathbf{C}_{1} & & & & \\
\mathbf{D}_{1} & \mathbf{C}_{2} & & & \mathbf{0} \\
& \mathbf{D}_{2} & \mathbf{C}_{3} & & \\
& & \ddots & \ddots & \\
& & & \mathbf{D}_{N-1} & \mathbf{C}_{N}
\end{array}\right]
$$

where $\mathbf{C}_{i}$ is a $K \times K$ lower triangular and $\mathbf{D}_{i}$ is a $K \times K$ upper triangular matrix. From (8) and (9), the following recursive relations can be deduced

$$
\begin{aligned}
\mathbf{C}_{1} \mathbf{C}_{1}^{T} & =\mathbf{H}_{0}(1) \\
\mathbf{C}_{i} \mathbf{D}_{i}^{T} & =\mathbf{H}_{1}^{T}(i), \quad \text { for } 1 \leq i \leq N-1 \\
\mathbf{C}_{i+1} \mathbf{C}_{i+1}^{T} & =\mathbf{H}_{0}(i+1)-\mathbf{D}_{i} \mathbf{D}_{i}^{T}, \quad \text { for } 1 \leq i \leq N-1 .
\end{aligned}
$$

As for $\mathbf{R}_{N}$, the Cholesky decomposition $\mathbf{R}_{M}=\mathbf{L}_{M} \mathbf{L}_{M}^{\mathrm{T}}$ exists, where $\mathbf{L}_{M}$ has the same structure as $\mathbf{L}$ except for the size and has $\hat{\mathbf{C}}_{j}$ and $\hat{\mathbf{D}}_{j}(1 \leq j \leq M-1)$ as the $j$ th blocks on its main diagonal and the first lower subdiagonal, respectively. Consequently, recursive relations similar to those in (10a)-(c) hold for $\hat{\mathbf{C}}_{j}, \hat{\mathbf{D}}_{j}$, and the corresponding blocks in $\mathbf{R}_{M}$. Using these recursive formulas, efficient algorithms for updating the Cholesky factors and user detection can be found [10].

It is noteworthy to point out that the Cholesky-factorizationbased algorithms for the OWD detector could also be used to solve the implementation problems of an exact FIR decorrelator. Assume that the decision delay of an exact FIR decorrelator is $p=(M-1) / 2$ where $M$ is the window length. Within a processing window, each information bit can be deemed to originate from a different synchronous user. In doing so, an asynchronous system is interpreted as an equivalent synchronous system. Let $\mathbf{S}$ be the matrix collecting all the user codes (discrete signature signals) of virtual synchronous users within a processing window. If all the user codes are linearly independent of each other, an exact decorrelating solution exists and the coefficient vector of the FIR decorrelator for the $j$ th user is the $(J p+j)$ th column of matrix $\mathbf{S} \mathbf{R}^{-1}$, where $\mathbf{R}=\mathbf{S}^{\mathrm{T}} \mathbf{S}$. Clearly, updating the coefficient vector directly is computationally not feasible. Note that $\mathbf{R}$ is equivalent to $\mathbf{R}_{N}$ as defined in (2) with $N$ equal to $M$. This suggests an efficient implementation as follows. Instead of using one long FIR decorrelating filter, we can employ three short-length matched filters for each user at the front end, which are matched to the entire user code, the earlier portion of the user code, and latter portion of the user code, respectively. An immediate advantage of doing so is that the updating of the receiver due to changes of the system profile becomes easier. If the changes are due to user activation/deactivation, the matched filters do not need to be updated. Collecting the outputs of the matched filters in a column vector $\mathbf{X}$, the soft output of the detector can then be written as $\mathbf{R}^{-1} \mathbf{x}$. Hence Cholesky-factorization-based algorithms can be used to efficiently update and invert $\mathbf{R}$. Moreover, the performance of an exact FIR decorrelator can be related to the decay rate of the impulse response of the IIR decorrelator and the method for window-length determination can also be used for the selection of the length of the FIR decorrelator.

The proposed detector offers some advantages over an exact FIR decorrelator. First, the OWD detector needs only one matched filter for each user while the latter needs three. Second, the OWD detector uses detected information bits to subtract the left-edge effects and treats the interference from the right edge as Gaussian noise. Consequently, the noise enhancement effect of an exact decorrelating solution is, to some degree, reduced and the performance of the OWD detector is expected to be slightly better than that of an exact FIR decorrelator for the same window length. This will become apparent from our analysis and simulation results in Section IV.

\section{B. Decay Rate of Impulse Response}

We now provide an analysis on the decay rate of the impulse response of the ideal decorrelating detector, which facilitates the determination of the required window length for the OWD detector.

Consider the ideal decorrelating detector as a multiple-input multiple-output (MIMO) discrete-time system with impulse response $\mathbf{F}(m, n) \in \mathcal{R}^{K \times K}$ whose $(i, j)$ th entry is the impulse response that relates the $j$ th user to the $i$ th user [12]. For the ideal decorrelating detector, $\mathbf{F}(m, n)$ is the $(m, n)$ th block of the linear mapping $\mathbf{R}_{N}^{-1}$. Since $\mathbf{L}$ is lower triangular, $\mathbf{L}^{-1}=$ $\left\{\mathbf{P}_{i j} \in \mathcal{R}^{K \times K}, 1 \leq i, j \leq N\right\}$ is also lower triangular. From (9) and the fact that $\mathbf{L}^{-1} \mathbf{L}=\mathbf{I}$, the $(i, j)$ th block $\mathbf{P}_{i j}$ with $i \geq j$ can be recursively found and is given by

$$
\mathbf{P}_{i j}=(-1)^{i-j} \mathbf{C}_{i}^{-1} \prod_{k=1}^{i-j} \mathbf{M}_{i-k}, \quad \text { for } i \geq j
$$

where $\mathbf{M}_{i}=\mathbf{D}_{i} \mathbf{C}_{i}^{-1}$. Equation (11) in conjunction with $\mathbf{R}_{N}^{-1}=\mathbf{L}^{-\mathrm{T}} \mathbf{L}^{-1}$ leads to

$\mathbf{F}(m, n)= \begin{cases}\sum_{j=m}^{N} \mathbf{P}_{j m}^{\mathrm{T}} \mathbf{P}_{j n} & \text { for } m=n \\ (-1)^{m-n} \mathbf{F}(m, m) \prod_{k=1}^{m-n} \mathbf{M}_{m-k} & \text { for } m>n \\ (-1)^{n-m} \prod_{k=m}^{n-1} \mathbf{M}_{k}^{\mathrm{T}} \mathbf{F}(n, n) & \text { for } n>m .\end{cases}$

Note that $\mathbf{M}_{i}=\mathbf{H}_{1}(i) \mathbf{K}_{i}^{-1}$ and $\mathbf{H}_{1}(i)$ may vary randomly with $i$, where $\mathbf{K}_{i}=\mathbf{C}_{i} \mathbf{C}_{i}^{\mathrm{T}}$. Although the exact decay rate of the impulse response of the ideal decorrelating detector for time-dependent systems is rather difficult to obtain, it was shown in [6] that the impulse response in a time-dependent system decays on the average faster than in a time-invariant system, given that the two systems have similar cross-correlation properties among the signature signals. A similar observation was also made on the impulse response of the noise whitening filter for a CDMA system [9]. Consequently, one can obtain an approximate upper bound of the decay rate of the impulse response in a time-dependent system by analyzing a time-invariant system. In what follows, we analyze the decay behavior of time-invariant systems.

For a time-invariant system, it was shown in [3] that as $N \rightarrow$ $\infty$, the ideal decorrelating detector tends to assume the form of a $K$-input $K$-output linear time-invariant (LTI) IIR filter. Therefore, as $i \rightarrow \infty, \mathbf{C}_{i}, \mathbf{D}_{i}$, and $\mathbf{K}_{i}=\mathbf{C}_{i} \mathbf{C}_{i}^{\mathrm{T}}$ converge to their 
limits $\mathbf{C}, \mathbf{D}$, and $\mathbf{K}$, respectively. A necessary and sufficient condition for the stability of the LTI IIR filter was obtained via a frequency-domain approach in [4]. For the purpose of our analysis, we give a necessary and sufficient condition for the stability in terms of the Cholesky factors of $\mathbf{R}_{N}$ as follows:

Proposition 1: The ideal LTI decorrelating filter is stable if and only if the limiting $\mathbf{C}$ is nonsingular and the spectral radius of $\mathbf{M}=\mathbf{D C}^{-1}$, denoted as $\rho(\mathbf{M})$, is strictly less than 1 .

Proof: Assuming that $\mathbf{C}$ is nonsigular, then $\mathbf{C}^{-1}$ exists and the impulse response of the LTI decorrelating filter can be written as

$$
\begin{gathered}
\mathcal{A}_{k}=\left(\mathcal{A}_{-k}\right)^{\mathrm{T}}=\lim _{\substack{n \rightarrow \infty \\
N \rightarrow \infty}} \mathbf{F}(n+k, n)=(-1)^{k} \mathcal{A}_{0} \mathbf{M}^{k}, \\
\text { for } k \geq 0
\end{gathered}
$$

where

$$
\mathcal{A}_{0}=\sum_{i=0}^{\infty}\left[\left(\mathbf{C}^{-1} \mathbf{M}^{i}\right)^{\mathrm{T}}\left(\mathbf{C}^{-1} \mathbf{M}^{i}\right)\right]
$$

Since $\left\|\mathbf{C}^{-1}\right\|$ is bounded, there exist positive constants $c_{1}$ and $c_{2}$ such that

$c_{1} \max _{1 \leq i, j \leq K}\left(\mathbf{M}^{k}\right)_{i j} \leq \max _{1 \leq i, j \leq K}\left(\mathbf{C}^{-1} \mathbf{M}^{k}\right)_{i j} \leq c_{2} \max _{1 \leq i, j \leq K}\left(\mathbf{M}^{k}\right)_{i j}$

where $(\cdot)_{i j}$ stands for the $(i, j)$ th entry of the matrix inside the bracket. For a given $\epsilon>0$, there is a constant $c$ such that

$$
\left|\left(\mathbf{M}^{k}\right)_{i j}\right| \leq c(\rho(\mathbf{M})+\epsilon)^{k}
$$

for all $k>0$ and $i, j=1,2, \ldots, K$ [11, pp. 298-299]. From (15) and (16), it can be shown that $\mathcal{A}_{0}$ is finite if and only if $\rho(\mathbf{M})<1$. Assuming that $\rho(\mathbf{M})<1$, by (16) we have

$$
c_{3} \rho^{k}(\mathbf{M}) \leq\left|\max _{1 \leq i, j \leq K}\left(\mathcal{A}_{k}\right)_{i j}\right| \leq c_{4}[\rho(\mathbf{M})+\epsilon]^{k}
$$

for any $k>0$, where $c_{3}$ and $c_{4}$ are positive constants. Since $\rho(\mathbf{M})<1,(17)$ implies that the impulse response $\left(\mathcal{A}_{k}\right)_{i j}$ of the $(i, j)$ th subsystem is absolutely summable. The sufficiency now follows from the fact that a MIMO system is stable if and only if every subsystem is stable. To prove the necessity, we assume that the LTI filter is stable. If so, matrix $\mathbf{C}$ must be nonsingular and (15) holds. Since $\mathcal{A}_{0}$ is finite for a stable filter, $\rho(\mathbf{M})$ must be less than 1 .

As an immediate consequence of (17), we can state the following proposition.

Proposition 2: If the LTI decorrelating filter is stable, its impulse response decays with an asymptotic decay rate $\rho(\mathbf{M})$.

It follows that if $\rho(\mathbf{M})<1$ is small, then the impulse response of the LTI decorrelating filter decays rapidly. Indeed, as will be demonstrated by our numerical examples in Section VI, $\rho(\mathbf{M})$ is usually much smaller than 1 for systems with moderate cross-correlation properties among the signature signals. It is also interesting to note that the convergence rate of $\mathbf{K}_{i}$ to $\mathbf{K}$ is linear with $\rho^{2}(\mathbf{M})$ as shown in Appendix I. By (10a)-(10c), fast convergence of $\mathbf{K}_{i}$ implies fast convergence of $\mathbf{M}_{i}$. Therefore, the decay rate of the impulse response of the OWD detector is approximately equal to $\rho(\mathbf{M})$ for a sufficiently large window length $M$.

\section{Determination of Window Length}

We now turn our attention to the determination of the window length. Denoting the first $k$ elements of vector $\mathbf{y}$ as $[\mathbf{y}]_{1: k}$, then $\left[\tilde{\mathbf{b}}_{i}\right]_{1: k K}$ contains the estimates of the user information bits within the first $k$ transmission intervals in $\tilde{\mathbf{b}}_{i}$. The difference between $\left[\hat{\mathbf{b}}_{i}\right]_{1:(p+1) K}$ in (6) and $\left[\tilde{\mathbf{b}}_{i}\right]_{1:(p+1) K}$ in (5) can be written as

$$
\begin{aligned}
& {\left[\mathbf{R}_{M}^{-1} \mathbf{r}_{e}\right]_{1:(p+1) K}=\left\{[\hat{\mathbf{F}}(1, M) \mathbf{x}]^{\mathrm{T}} \quad[\hat{\mathbf{F}}(2, M) \mathbf{x}]^{\mathrm{T}}\right.} \\
& \left.\cdots \quad[\hat{\mathbf{F}}(p+1, M) \mathbf{x}]^{\mathrm{T}}\right\}^{\mathrm{T}}
\end{aligned}
$$

where $\mathbf{x}=\mathbf{H}_{1}(i+p+1)^{\mathrm{T}} \mathbf{E}(i+p+1) \mathrm{b}(i+p+1)$ and $\hat{\mathbf{F}}(n, M) \in \mathcal{R}^{K \times K}$ is the $(n, M)$ th block of $\mathbf{R}_{M}^{-1}$, and can be interpreted as the impulse response of the OWD detector. Replacing $N$ in (12) with $M$, we have

$$
\hat{\mathbf{F}}(n, M)=(-1)^{M-n}\left(\prod_{k=n}^{M-1} \hat{\mathbf{M}}_{k}^{\mathrm{T}}\right) \hat{\mathbf{K}}_{M}^{-1}
$$

where $\hat{\mathbf{M}}_{k}=\hat{\mathbf{D}}_{k} \hat{\mathbf{C}}_{k}^{-1}$ and $\hat{\mathbf{K}}_{k}=\hat{\mathbf{C}}_{k} \hat{\mathbf{C}}_{k}^{\mathrm{T}}$. Denoting $\hat{\mathbf{F}}(n, M) \mathbf{H}_{1}^{\mathrm{T}}(i+p+1)$ as $\tilde{\mathbf{F}}_{n}$, then (19) in conjunction with $\mathbf{M}_{M}=\mathbf{H}_{1}(i+p+1) \mathbf{K}_{M}^{-1}$ leads to

$$
\tilde{\mathbf{F}}_{n}=(-1)^{M-n} \prod_{k=n}^{M} \hat{\mathbf{M}}_{k}^{\mathrm{T}}
$$

The right-edge effect $\mathbf{r}_{e}$ can be neglected if $\left\|\tilde{\mathbf{F}}_{n}\right\|_{\infty}$ for $n \leq$ $p+1$ is sufficiently small, where $\|\cdot\|_{\infty}$ denotes the maximum infinity norm [11]. Since the impulse response of the detector decays $\left\|\tilde{\mathbf{F}}_{p+1}\right\|_{\infty}$ is in general larger than $\left\|\tilde{\mathbf{F}}_{n}\right\|_{\infty}$ for $p+1>n$; hence the window length can be determined as the smallest $M$ such that

$$
\left\|\tilde{\mathbf{F}}_{p+1}\right\|_{\infty}<\epsilon
$$

where $\epsilon>0$ is a prescribed tolerance. In the next section, it will be shown that $\epsilon$ in a practical system can be selected such that $\epsilon \sqrt{\kappa} \ll 1$, where $\kappa$ is the maximum difference among the received user energies.

For systems with constant correlations within the working window and for sufficiently large $p$, the fast convergence of $\hat{\mathbf{M}}_{k}$ implies that $\hat{\mathbf{M}}_{k} \approx \hat{\mathbf{M}}_{p+1}$ if $k>p+1$. Hence, (20) leads to

$$
\left\|\tilde{\mathbf{F}}_{p+1}\right\|_{\infty}<c\left[\rho\left(\hat{\mathbf{M}}_{p+1}\right)\right]^{p+1} .
$$

In a practical implementation, constant $c$ in (22) can be simply taken as $\left\|\hat{\mathbf{M}}_{p+1}\right\|_{\infty} / \rho\left(\hat{\mathbf{M}}_{p+1}\right)$, and $\rho\left(\hat{\mathbf{M}}_{p+1}\right)$ can be estimated using various numerical methods such as the power method [11]. For slowly varying systems where correlations remain approximately unchanged within a working window, the criterion in (21) in conjunction with (22) can be used to determine the window length when the OWD detector needs to be updated. 


\section{Performance Analysis}

In order to determine the window length, or equivalently the tolerance $\epsilon$ in (21), we need two performance measures, namely, the asymptotic multiuser efficiency (AME) and the near-far (NF) resistance. However, an exact performance formula is difficult to obtain because the OWD detector uses the reconstructed information bits to correct the left-edge effect (i.e., the MAI at the beginning of the window). Hence we assume a perfect left-edge correction. This assumption leads to accurate estimates for the exact performance measures since the error due to a wrong left-edge correction does not accumulate, as discussed before. Since the most inaccurate estimate of user bits usually occurs in the central transmission interval of a working window, we only discuss the user bits in this interval as a worst case study.

The AMEcharacterizes the performance degradation due to the MAI in a vanishing noise case [1]. Based on the above assumptions, the AME of the $i$ th bit of the $k$ th user can be deduced as

$$
\begin{aligned}
\eta_{k}(i)=\max ^{2} & \left\{0, \frac{1}{\sqrt{e_{k}(i)}}\right. \\
& \left.\times \min _{\substack{\mathbf{b}(i+p+1) \in\{-1,1\}^{K} \\
b_{k}(i)=1}} \frac{\sqrt{e_{k}(i)}+\nu[\mathbf{b}(i+p+1)]}{\sqrt{d_{p K+k}}}\right\} .
\end{aligned}
$$

The NF resistance of the $i$ th bit of the $k$ th user is defined as its worst case AME over all possible energies of the other information bits. According to this definition, the OWD cannot be NF resistance in the strict sense. However, given that the received power is finite in practice, the concept of the power-limited NF resistance introduced in [6] applies, and the power-limited NF resistance of the $i$ th bit of the $k$ th user is given by

$$
\bar{\eta}_{k}(i)=\inf _{\substack{0 \leq e_{l}(j) \leq e_{\max }(j) \\(l, j) \neq(k, i)}} \eta_{k}(i)
$$

where $e_{\max }(j)=\max _{1 \leq l \leq K} e_{l}(j)$. It follows that

$$
\bar{\eta}_{k}(i)=\max ^{2}\left\{0, \frac{1-\mu \sqrt{\frac{e_{\max }(i+p+1)}{e_{k}(i)}}}{\sqrt{d_{p K+k}}}\right\}
$$

where $\mu$ is the $l_{1}$ norm [11] of the $k$ th row of $\mathbf{F}(p+1, M) \mathbf{H}_{1}(i+$ $p+1)^{\mathrm{T}}$. In mobile CDMA communications, the received power imbalance, which is defined as

$$
\kappa=\max _{1 \leq j \leq N} \frac{e_{\max }(j)}{e_{\min }(j)}
$$

where $e_{\min }(j)=\min _{1 \leq l \leq K} e_{l}(j)$, serves as a performance measure of power control. If $\left\|\mathbf{F}(p+1, M) \mathbf{H}_{1}(i+p+1)^{\mathrm{T}}\right\|_{\infty}<\epsilon$, a lower bound for $\bar{\eta}=\min _{k, i} \bar{\eta}_{k}(i)$ can be deduced as

$$
\bar{\eta} \geq \max ^{2}\left\{0, \frac{1-\epsilon \sqrt{\kappa}}{\sqrt{d_{p K+k}}}\right\} \text {. }
$$

This can serve as a guide for the selection of tolerance $\epsilon$ for the determination of the window length.

We conclude this section with an observation concerning the performance of the OWD detector relative to that of the ideal decorrelating detector. It can be seen from (23) and (27) that if $\epsilon$ is selected such that $\epsilon \sqrt{\kappa} \ll 1$, then the AME and the power-limited NF resistance of the proposed detector is approximately $1 / d_{p K+k}$. In [3], the AME of the ideal decorrelating detector was derived as $1 / d_{(i-1) K+k}^{\text {ideal }}$, where $1 / d_{k}^{\text {ideal }}$ denotes the $(k, k)$ th entry of $\mathbf{R}_{N}^{-1}$. Since $\mathbf{R}_{M}$ is a principal submatrix of $\mathbf{R}_{N}$, we have $1 / d_{p K+k} \geq 1 / d_{(i-1) K+k}^{\text {ideal }}$ [11]. This shows that in some cases the performance of the OWD detector can even be better than that of the ideal detector. This feature is a consequence of the fact that the output of the OWD detector is independent of the MAI from outside the working window and hence an improved signal-to-noise ratio is achieved.

\section{NUMERICAL EXAMPLES}

We first consider a two-user time-invariant system. The simplicity of the system allows us to carry out an analysis on issues such as the convergence of $\mathbf{K}_{i}$ and the system performance in an explicit manner. Assuming that $\left(\mathbf{H}_{0}\right)_{12}=r_{1}$ and $\left(\mathbf{H}_{1}\right)_{12}=r_{2}$, then $\left|r_{1}\right|+\left|r_{2}\right| \leq 1$. By (10a)-(c), the spectral radius of $\mathbf{M}_{i}$, denoted as $\rho_{i}$, can be computed by using the recursive formula

$$
\rho_{i}=\left|\frac{r_{1} r_{2}}{x_{i}\left(\frac{1-r_{1}^{2}}{x_{i}}\right)}\right|
$$

where $x_{i+1}=1-r_{2}^{2} /\left(x_{i}-r_{1}^{2}\right)$ and $x_{1}=1$. This leads to

$$
x=\lim _{i \rightarrow \infty} x_{i}=\frac{1+r_{1}^{2}-r_{2}^{2}+\sqrt{\left(1+r_{1}^{2}-r_{2}^{2}\right)^{2}-4 r_{1}^{2}}}{2} .
$$

Letting $i \rightarrow \infty$ in (28) and substituting (29) into (28) gives $\rho=2\left|r_{1} r_{2}\right| /\left(\tau+\sqrt{\tau^{2}-2 r_{1}^{2} r_{2}^{2}}\right)$ with $\tau=1-r_{1}^{2}-r_{2}^{2}$. Note that $\rho=1$ if $\left|r_{1}\right|+\left|r_{2}\right|=1$, which corresponds to an unstable realization of the ideal decorrelating detector. The plot shown in Fig. 1 depicts $\rho$ versus $r_{1}$ and $r_{2}$. It can be seen that $\rho$ is usually quite small. Specifically, we have $\rho<0.4$ if $\left|r_{1}\right|+\left|r_{2}\right|<0.9$ whereas $\rho<0.25$ if $\left|r_{1}\right|+\left|r_{2}\right|<0.8$. The convergence rate of $\rho_{i}$, given by the smallest value of $i$ such that $\left|\rho_{i}-\rho\right| / \rho<0.01$, and the smallest window length given by (21) with $\epsilon=0.01$ are given in Table I. We see that even in the cases where the cross-correlation properties are much poorer than what is often encountered in practice, the convergence rate of $\rho_{i}$ remains high and the window length required is moderate.

We now consider systems with multiple users. To demonstrate the performance of the proposed detector, extensive computer simulations were conducted. In all the simulations, spreading signals with a rectangular chip waveform were selected from a family of the 127-chip Gold code.

To study the NF resistance of the OWD detector, the energy received from the desired user was assumed to be $e_{\text {min }}$. The power-limited NF resistance achieved for time-invariant systems under various conditions is illustrated in Fig. 2. It is observed that the loss in the NF resistance increases with the 


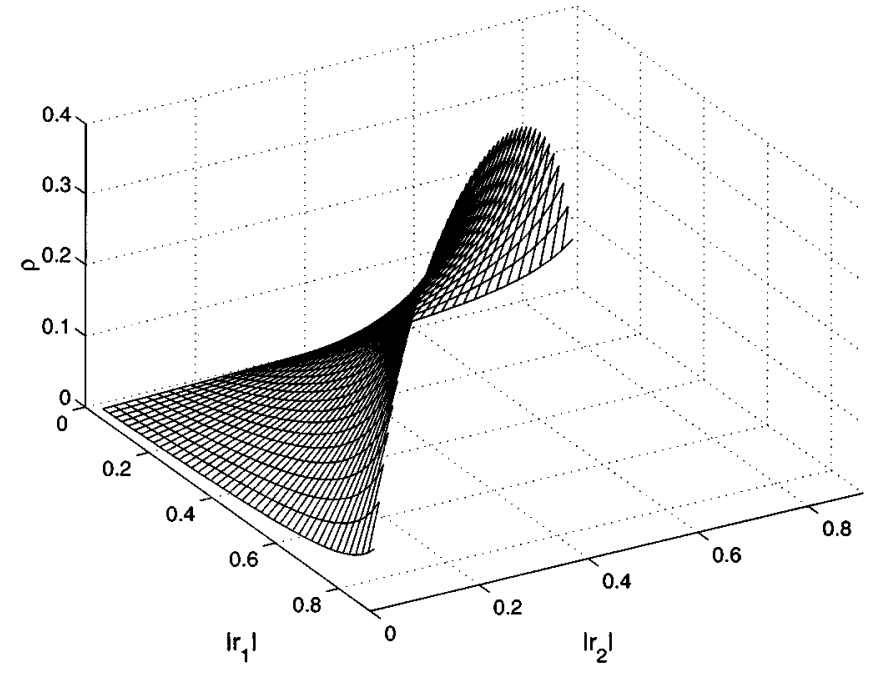

Fig. 1. Spectral radius of $\mathbf{M}$ in a two-user system.

TABLE I

CONVERGENCE RATE OF $\rho_{i}$ /SMALLEST Window Length Given BY (21) WITH $\epsilon=0.01$ IN A TWO-USER SySTEM

\begin{tabular}{c|cccccccc}
$r_{1} \backslash r_{2}$ & 0.1 & 0.2 & 0.3 & 0.4 & 0.5 & 0.6 & 0.7 & 0.8 \\
\hline 0.1 & $2 / 5$ & $2 / 5$ & $2 / 5$ & $2 / 5$ & $2 / 5$ & $2 / 5$ & $3 / 5$ & $3 / 7$ \\
0.2 & $2 / 5$ & $2 / 5$ & $2 / 5$ & $2 / 7$ & $2 / 7$ & $3 / 7$ & $3 / 11$ & \\
0.3 & $2 / 5$ & $2 / 5$ & $2 / 7$ & $2 / 7$ & $3 / 7$ & $3 / 11$ & & \\
0.4 & $2 / 5$ & $2 / 7$ & $2 / 7$ & $3 / 9$ & $3 / 11$ & & & \\
0.5 & $2 / 5$ & $2 / 7$ & $2 / 9$ & $3 / 11$ & & & & \\
0.6 & $2 / 5$ & $2 / 7$ & $3 / 11$ & & & & & \\
0.7 & $2 / 7$ & $3 / 11$ & & & & & & \\
0.8 & $2 / 9$ & & & & & & &
\end{tabular}

number of users and the received power imbalance. The simulation results indicate that for a system with 20 users and a received power imbalance $e_{\max } / e_{\min }$ of $10 \mathrm{~dB}$, a window length of 3 or 5 is usually sufficient to yield a performance close to that of the ideal decorrelating detector.

The overall bit-error probability (BEP) of the OWD detector for time-invariant systems over an AWGN channel is plotted in Fig. 3. Here, the user received energies were uniformly distributed on interval $\left[e_{\min }, e_{\max }\right]$. The performance of the detector under two conditions, i.e., using correct bits as feedback and using detected bits as feedback, is also compared in the figures. As is expected, no apparent differences in the performance are observed under these two conditions. It can also be observed form the figure that the performance of the OWD detector with a window-length 5 is close to that of the ideal decorrelating detector and sometimes it can even be slightly better.

\section{CONCLUSIONS}

The convergence of the ideal decorrelating detector to its limiting IIR filter and the decay of its impulse response have been studied. Based on these two useful properties, an efficient overlapping-window decorrelating detector for DS-CDMA systems has been proposed. Then, a signal-adapted criterion for the determination of the window length has been developed. This criterion enables the OWD detector to select the smallest window

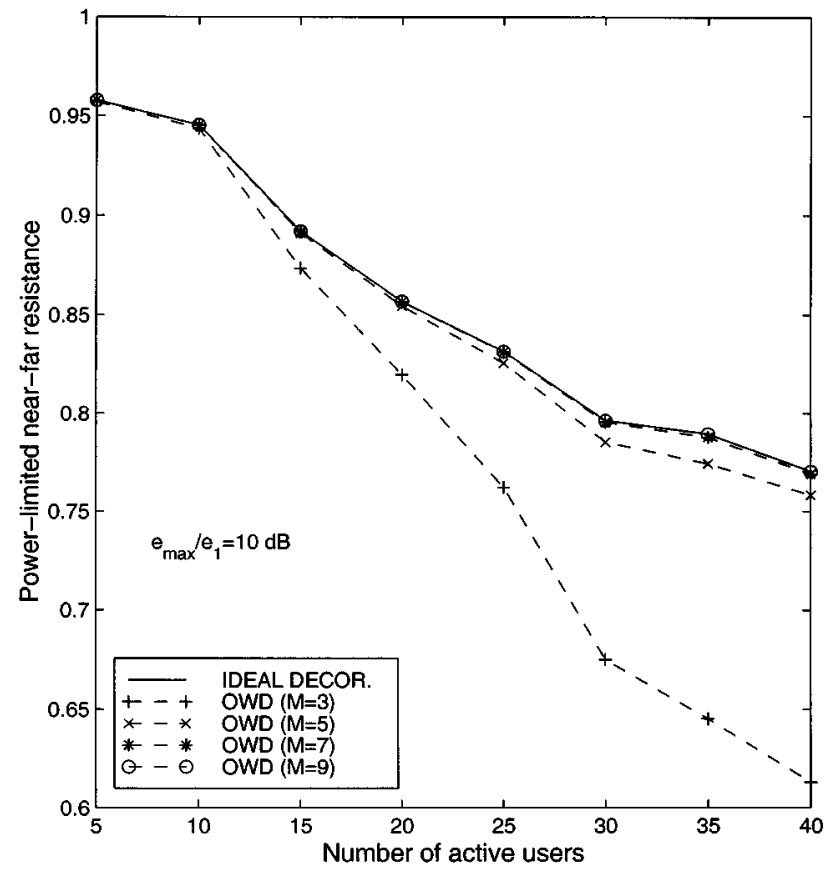

(a)

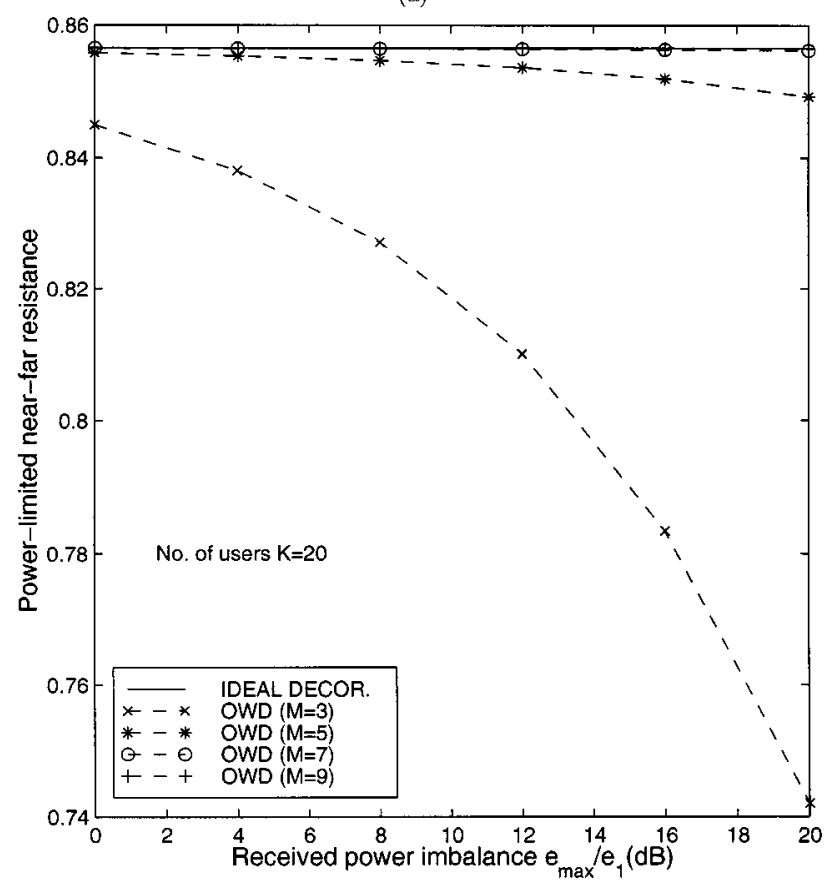

(b)

Fig. 2. Power-limited NF resistance for user 1 in a time-invariant system (a) as a function of the number of active users and (b) the received power imbalance.

length, and hence the smallest processing delay, to achieve a prescribed NF resistance under a given near-far situation. A theoretical analysis as well as simulation results have demonstrated that the proposed OWD detector with a small to moderate window length offers performance close to or even better than that of the ideal decorrelating detector.

The proposed detection scheme and analysis on the behavior of the ideal decorrelating detector can be easily extended to the case of the linear minimum mean-squared error multiuser detector. 


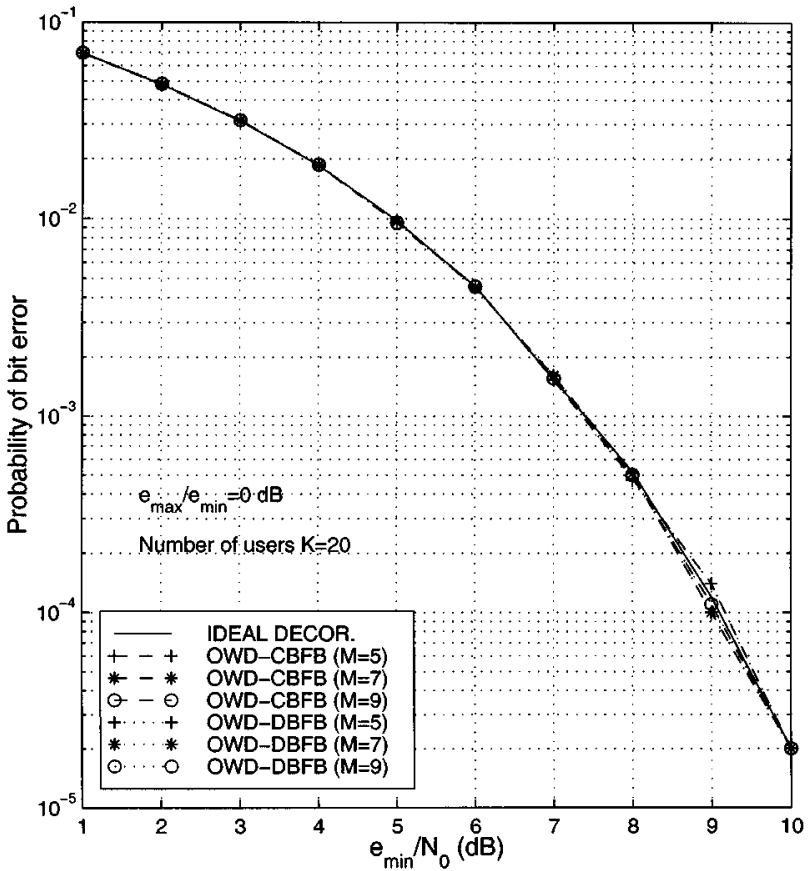

(a)

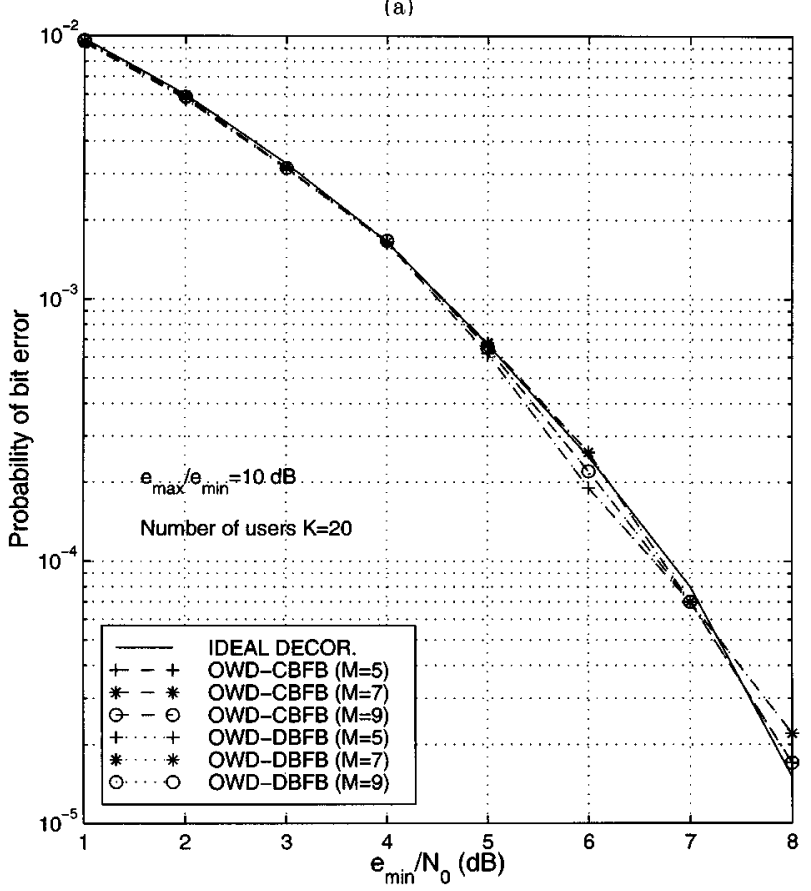

(b)

Fig. 3. Comparison of bit-error probabilities under the conditions of using correct bits as feedback (CBFB) and using detected bits as feedback (DBFB) over an AWGN channel. (a) Received power imbalance $e_{\max } / e_{\min }=0 \mathrm{~dB}$. (b) Received power imbalance $e_{\max } / e_{\min }=10 \mathrm{~dB}$.

\section{APPENDIX \\ CONVERGENCE OF $\mathbf{K}_{i}$}

For systems with time-invariant correlations, (10c)implies that

$$
\mathbf{K}_{i+1}=\mathbf{H}_{0}-\mathbf{H}_{1} \mathbf{K}_{i}^{-1} \mathbf{H}_{1}^{\mathrm{T}}, \quad \text { for } 1 \leq i \leq N-1
$$

where $\mathbf{K}_{1}=\mathbf{H}_{0}$ and $\mathbf{K}_{i}$ is positive definite. It follows that

$$
\mathbf{K}_{i+1}-\mathbf{K}_{i}=\mathbf{H}_{1} \mathbf{K}_{i}^{-1}\left(\mathbf{K}_{i}-\mathbf{K}_{i-1}\right) \mathbf{K}_{i-1}^{-1} \mathbf{H}_{1}^{\mathrm{T}}
$$

By repeating (A.2) in conjunction with (10a)-(c), we obtain

$$
\mathbf{K}_{i+1}-\mathbf{K}_{i}=\prod_{j=0}^{p-1} \mathbf{M}_{i-j} \cdot\left(\mathbf{K}_{i+1-p}-\mathbf{K}_{i-p}\right) \cdot \prod_{n=i-p}^{i-1} \mathbf{M}_{n}
$$

Hence

$$
\frac{\left\|\mathbf{K}_{i+1}-\mathbf{K}_{i}\right\|}{\left\|\mathbf{K}_{i+1-p}-\mathbf{K}_{i-p}\right\|} \leq\left\|\prod_{j=0}^{p-1} \mathbf{M}_{i-j}\right\|\left\|\prod_{n=i-p}^{i-1} \mathbf{M}_{n}\right\| .
$$

Now if $i-p$ is sufficiently large such that $\mathbf{K}_{i-p} \approx \mathbf{K}$ and $\mathbf{M}_{i-p} \approx \mathbf{M}$, we have

$$
\left\|\prod_{j=0}^{p-1} \mathbf{M}_{i-j}\right\|\left\|\prod_{n=i-p}^{i-1} \mathbf{M}_{n}\right\| \approx\left\|\mathbf{M}^{p}\right\|^{2}
$$

It can be shown that there exists a constant $c$ such that $\left\|\mathbf{M}^{p}\right\| \leq$ $c\left[\rho^{p}(\mathbf{M})+\epsilon\right]$ for a given $\epsilon>0$ [11], which in conjunction with (A.4) and (A.5) gives

$$
\frac{\left\|\mathbf{K}-\mathbf{K}_{i+1}\right\|}{\left\|\mathbf{K}-\mathbf{K}_{i}\right\|} \leq c_{1}[\rho(M)+\epsilon]^{2} .
$$

This implies that $\mathbf{K}_{i}$ converges linearly with $\rho^{2}(\mathbf{M})$ when $\mathbf{K}_{i}$ is close to $\mathbf{K}$.

\section{REFERENCES}

[1] S. Verdú, "Minimum probability of error for asynchronous gaussian multiple-access channels," IEEE Trans. Inform. Theory, vol. IT-32, pp. 85-96, Jan. 1986.

[2] S. Moshavi, "Multi-user detection for DS-CDMA communications," IEEE Commun. Mag., vol. 34, pp. 124-136, Oct. 1996.

[3] R. Lupas and S. Verdú, "Near-far resistance of multiuser detectors in asynchronous channels," IEEE Trans. Commun., vol. 38, pp. 725-736, Apr. 1990.

[4] Z. Xie, R. T. Short, and C. K. Rushforth, "A family of suboptimal muti-user detectors," IEEE J. Select. Areas Commun., vol. 8, pp. 683-90, May 1990.

[5] S. S. H. Wijayasuria, G. H. Norton, and J. P. McGeehan, "A sliding window decorrelating receiver for multiuser DS-CDMA mobile radio networks," IEEE Trans. Veh. Technol., vol. 45, pp. 503-521, Aug. 1996.

[6] M. J. Juntti and B. Aazhang, "Finite memory-length linear multiuser detection for asynchronous CDMA communications," IEEE Trans. Commun., vol. 45, pp. 611-622, May 1997.

[7] F. Zheng and S. K. Barton, "Near far resistant detection of CDMA signals via isolation bit insertion," IEEE Trans. Commun., vol. 43, pp. 1313-1317, Feb./Mar./Apr. 1995.

[8] M. K. Tsatsanis and G. B. Giannakis, "Optimal decorrelating receivers for DS-CDMA systems: a signal processing framework," IEEE Trans. Signal Processing, vol. 44, pp. 3044-3055, Dec 1996.

[9] L. Wei and K. Rasmussen, "A near ideal noise whitening filter for an asynchronous time-varying CDMA system," IEEE Trans. Commun., vol. 43, pp. 1313-1317, Feb./Mar./Apr 1995.

[10] X. F. Wang, W.-S. Lu, and A. Antoniou, "Efficient implementation of linear multiuser detectors," Proc. ICASSP'98, vol. 6, pp. 3221-3224.

[11] R. A. Horn and C. R. Johanson, Matrix Analysis. Cambridge, U.K.: Cambridge Univ. Press, 1990.

[12] R. E. Crochiere and L. R. Rabiner, Multirate Digital Signal Processing. Englewood Cliffs, NJ: Prentice-Hall, 1983. 


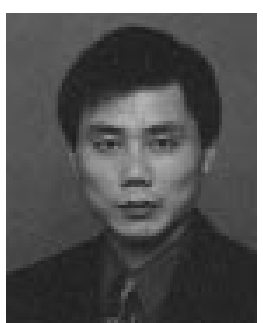

Xiaofeng Wang received the B.S. degree in electronics from Wuhan University, China, in 1991, and the M.E. degree in electronic engineering from Beijing University of Posts and Telecommunications, in 1994. Currently, he is working toward the Ph.D. degree with the Department of Electrical and Computer Engineering, University of Victoria, Victoria, BC, Canada.

Since February 2000, he has been working for PMC-Sierra Corporation. His research interests include digital signal processing for communications and broad-band network architectures.

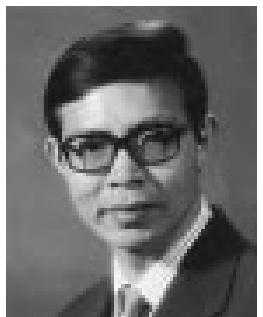

Wu-Sheng Lu (F'99) received the B.S. degree in mathematics from Fudan University, China, in 1964, and the M.S. degree in electrical engineering and the $\mathrm{Ph} . \mathrm{D}$. degree in control science from the University of Minnesota, Minneapolis, in 1983 and 1984, respectively.

He was a Postdoctoral Fellow at the University of Victoria, Victoria, BC, Canada, in 1985, and a Visiting Assistant Professor at the University of Minnesota, Minneapolis, in 1986. Since 1987, he has been with the University of Victoria where he is currently a Professor. His teaching and research interests include the areas of digital signal processing and numerical optimization. He is the co-author (with A. Antoniou) of Two-Dimensional Digital Filters (New York: Marcel Dekker, 1992).

Dr. Lu is a Fellow of the Engineering Institute of Canada. He was elected Fellow of the IEEE for contributions to multidimensional and multirate digital filters and discrete systems. He was an Associate Editor of the Canadian Journal of Electrical and Computer Engineering in 1989 and the Editor of the same journal from 1990 to 1992. He was an Associate Editor of IEEE TRANSACTIONS ON CIRCUITS AND SYSTEMS II from 1993 to 1995. Presently he serves as an Associate Editor for the IEEE TRANSACTIONS ON CIRCUITS AND SYSTEMS I and the International Journal of Multidimensional Systems and Signal.

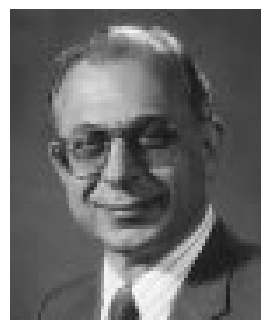

Andreas Antoniou (F'82) received the B.Sc. (Eng.) and Ph.D. degrees in electrical engineering from the University of London, London, U.K., in 1963 and 1966, respectively.

From 1966 to 1969 , he was Senior Scientific Officer at the Post Office Research Department, London, U.K., and from 1969 to 1970, he was a member of the Scientific Staff at the R \& D Laboratories of Northern Electric Company Ltd., Ottawa, ON, Canada. From 1970 to 1983, he served in the Department of Electrical and Computer Engineering. Concordia University, Montreal, PQ, Canada, as Professor from June 1973 and as Chairman from December 1977. He served as Founding Chairman of the Department of Electrical and Computer Engineering, University of Victoria, Victoria, BC, Canada, from July 1983 to June 1990 and is now Professor in the same department. His teaching and research interests include the areas of electronics, network synthesis, digital system design, active and digital filters, and digital signal processing. He published extensively in these areas. One of his papers on gyrator circuits was awarded the Ambrose Fleming Premium by the Institution of Electrical Engineers, UK. He is the author of Digital Filters: Analysis, Design, and Applications (New York: McGraw-Hill) and co-author (with W. -S. Lu) of Two-Dimensional Digital Filters (New York: Marcel Dekker).

Dr. Antoniou is a member of the Association of Professional Engineers and Geoscientists of B.C. and Fellow of the Institution of Electrical Engineers. He was elected Fellow of the Institute of Electrical and Electronics Engineers for contributions to active and digital filters, and to electrical engineering education. He served as Associate Editor of IEEE TRANSACTIONS ON CIRCUITS AND SYSTEMS from June 1983 to May 1985 and as Editor from June 1985 to May 1987, and was a member of the Board of Governors of the Circuits and Systems Society during 1995-1997. He was recently awarded a CAS Golden Jubilee Medal by the IEEE Circuits and Systems Society in recognition of outstanding achievements in the area of circuits and systems and the B.C. Science Council Chairman's Award for Career Achievement for 2000. 